\title{
Novel “After Minoxidil” Spray Improves Topical Minoxidil Compliance and Hair Style Manageability
}

Maja Kovacevic, John McCoy*, Jerry Shapiro, Rod Sinclair,
Sergio Vano Galvan, Mohamad Goldust, Mirna Situm, Andy
Goren
*Author whom correspondence should be addressed (johnm@appliedbiology.com)

Conflict of Interest: There is no conflict of interest.

\section{Author Affiliations:}

Maja Kovacevic, MD: Department of Dermatology and Venereology, University of Rome "G.Marconi", Rome, Italy.

John McCoy, PhD: Applied Biology, Irvine, California, USA.

Jerry Shapiro, MD: Department of Dermatology, New York University Langone Medical Center, New York City, New York, USA.

Rodney Sinclair, MD: Department of Medicine, University of Melbourne, Victoria, Australia.

Sergio Vaño-Galvan, MD, PHD. Trichology Unit, Dermatology Department, Ramon y Cajal Hospital, IRYCIS, University of Alcala, Madrid, Spain.

This is the author manuscript accepted for publication and has undergone full peer review but has not been through the copyediting, typesetting, pagination and proofreading process, which may lead to differences between this version and the Version of Record. Please cite this article as doi: 10.1111/JOCD.13630

This article is protected by copyright. All rights reserved 
Mohamad Goldust, MD: University of Rome G. Marconi, Rome, Italy; Department of Dermatology, University Hospital Basel, Basel, Switzerland

Mirna Situm,MD: Department of Dermatology and Venereology, University Hospital Center "Sestre milosrdnice", Zagreb, Croatia.

Andy Goren, MD: Applied Biology, Irvine, California, USA. Department of Dermatology and Venereology, Clinical Hospital Center Sestre Milosrdnice, Croatia.

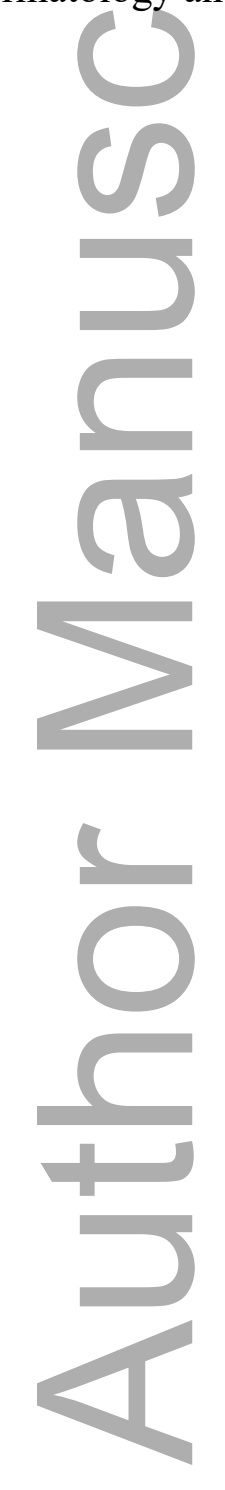

This article is protected by copyright. All rights reserved 
DR. MAJA KOVACEVIC (Orcid ID : 0000-0001-6579-5535)

DR. J. MCCOY (Orcid ID : 0000-0003-1577-9910)

DR. ANDY GOREN (Orcid ID : 0000-0002-8190-2289)

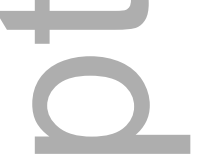

Article type : Original Contribution

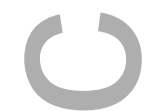

Novel “After Minoxidil" Spray Improves Topical Minoxidil Compliance and Hair Style Manageability

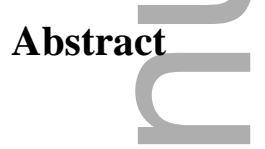

Topical minoxidil is the only US FDA drug approved for the treatment of female pattern hair loss (FPHL). While the safety profile of topical minoxidil is excellent, the efficacy of minoxidil in hair growth is extremely low. A recent survey of 8,000 people observed that only $4 \%$ of hair loss patients using an over-the-counter minoxidil were very satisfied with their results. In contrast, in clinical studies with an intervening physician, approximately $30-40 \%$ of patients demonstrate an appreciable benefit. Compliance with topical drug regimens is often a major obstacle, limiting their effectiveness. Topical minoxidil leaves a greasy residue on the hair, which is especially problematic for women who do not wash their hair daily. As such, we set out to develop an "After Minoxidil" companion spray to minoxidil that removes residual minoxidil from the hair, where it is not needed, yet leaves minoxidil on the scalp where it is required. We hypothesized that improving the cosmetic properties of minoxidil would improve patient compliance with the drug and subsequently improve clinical outcomes. In our cohort of FPHL patients, use of the novel "After Minoxidil" spray restored ease of styling and reduced greasiness to preminoxidil level in $65 \%$ and $85 \%$ of subjects, respectively. The average reduction in perceived greasiness was $78 \%$. Importantly, $70 \%$ of subjects interviewed stated they 
would likely continue to use the minoxidil and "After Minoxidil" treatment regimen for 6 months, versus $0 \%$ willing to use minoxidil alone.

\section{Introduction}

Female pattern hair loss (FPHL) also termed female androgenetic alopecia is a common condition affecting approximately $40 \%$ of women by the age of $60 .{ }^{1} 2 \%$ minoxidil solution and $5 \%$ minoxidil foam are the only US FDA approved drugs for the treatment of FPHL. In clinical trial, $5 \%$ minoxidil was shown to have superior efficacy to $2 \%$ minoxidil, however, the effectiveness of minoxidil, in general, is low (approximately $39 \%$ of subjects respond). ${ }^{2}$ According to a Consumers Reports survey of 8,000 people ${ }^{3}$, only $4 \%$ of respondents reported satisfaction with topical minoxidil for hair growth. In contrast, during clinical studies with an intervening physician, approximately $30-40 \%$ of patients respond to topical minoxidil treatment. ${ }^{4}$ The large disparity in patient satisfaction may be attributed to lower compliance with the over-the-counter treatment. For most topical drugs, including minoxidil, compliance with the daily treatment regimen is a major barrier that limits clinical efficacy. ${ }^{5}$ Dermatologists often report that many patients prescribed topical minoxidil stopped using the drug after only one month of use (personal communication). Because it takes at least 6 month to see appreciable results with topical minoxidil, we hypothesized that efficacy was not the main determining factor limiting patient compliance. Complaints about the aesthetic qualities of topical minoxidil have been reported. ${ }^{5-6}$ Greasy residue left on the hair is an especially negative quality for women, who do not wash their hair as frequently as men. As such, we set out to design a product that would remove minoxidil residue from hair while leaving the drug on the scalp. We theorized that an adjuvant product that would improve the cosmetic aesthetics of topical minoxidil, could greatly improve patient compliance with the treatment regimen, and subsequently improve its clinical efficacy.

\section{Methods}


Twenty women suffering from female pattern hair loss were recruited from an outpatient dermatology department at a hospital (Zagreb, Croatia). The average age of the subjects was 35 . Subjects were asked to style their hair normally prior to presenting to the hospital for the study. At baseline subjects were asked to rate how manageable their hair was on a Likert scale ( 1 being "very manageable" and 10 being "extremely difficult to manage"). Subjects also reported the ease of hair styling (rated 1-10, 1 being "easy to style" and 10 being "extremely difficult to style"). Additionally, subject were asked to rate the level of hair greasiness on a Likert scale ( 1 being "not greasy at all" and 10 being "extremely greasy"). After recording the baseline information, the principal investigator instructed the subjects to apply $1 \mathrm{~mL}$ of a $2 \%$ topical minoxidil solution to the frontoparietal scalp and massage the solution into the scalp. $2 \%$ solution was chosen because it is the US FDA approved solution dosage of topical minoxidil for women and 5\% foam was not available at the site of the study. Five minutes following application of topical minoxidil, each subject was asked to style their hair and subsequently report the ease of hair styling, ease of hair manageability, and hair greasiness. Subjects were asked if they would likely continue to use minoxidil for 90 days. After recording the information, the PI applied the novel "After Minoxidil" spray to the frontoparietal area. The "After Minoxidil" spray is a novel proprietary formulation containing a blend of aluminum starch octenylsuccinate, acrylates copolymer, and sodium bicarbonate; it is aerosolized and sprayed on the hair. The components of the "After Minoxidil" spray are designed to absorb lipophilic materials from the hair but the aerosolized material does not reach the scalp. Each subject was asked to style their hair and subsequently rate the ease of hair styling, ease of hair manageability, and hair greasiness. Subjects were asked if they would likely continue to use the minoxidil and "After Minoxidil" regimen for 90 days. For each subject, photos were obtained at each phase of the study and were assessed by a blinded independent expert. Data were tabulated as the average of the twenty women's ratings for each category; statistically significance the categories before and after application of the "After Minoxidil" product were calculated with a two-tailed student's t-test (MedCalc 19.3.1).

\section{Results}

This article is protected by copyright. All rights reserved 
Twenty subjects completed the study. The average score for each question in the survey are displayed in Figure 1. The average ease of styling score was 3.9 prior to applying minoxidil, 5.7 after applying minoxidil and 3.8 following "After Minoxidil" application. Ease of styling following "After Minoxidil" application was significantly better compared to after topical minoxidil application $(\mathrm{p}=0.002)$. 65\% (13/20) of subjects reported a score at or below their pre-minoxidil score after using the "After Minoxidil" product. The average hair manageability score was 4.75 prior to applying minoxidil, 6.2 after applying minoxidil and 4.75 following "After Minoxidil" application. Hair manageability following "After Minoxidil” application was significantly better compared to after topical minoxidil application $(\mathrm{p}=0.0049) .50 \%(10 / 20)$ of subjects reported a score at or below their pre-minoxidil score after using the "After Minoxidil" product. The average hair greasiness score was 6.4 prior to applying minoxidil, 8.65 after applying minoxidil and 4.85 following "After Minoxidil” application. Hair greasiness following "After Minoxidil" application was significantly lower compared to after topical minoxidil application $(\mathrm{p}<0.0001)$. 85\% $(17 / 20)$ of subjects reported a score at or below their preminoxidil score after using the "After Minoxidil" product. All subjects reported reduced hair greasiness following "After Minoxidil" application. Most notably, among the 20 subjects interviewed, $70 \%$ stated they would likely continue to use the minoxidil and “After Minoxidil" treatment regimen for 6 months, versus $0 \%$ willing to use minoxidil alone.

\section{Discussion}

Topical minoxidil is the only US FDA approved drug for female pattern hair loss (FPHL). While controlled clinical studies have demonstrated a clinical response in 30$40 \%$ of subjects ${ }^{4}$, over-the-counter consumer satisfaction is extremely low i.e., $4 \%{ }^{3}$ Consumer satisfaction with topical minoxidil may be attributable to low compliance. To observe clinical efficacy of topical minoxidil for FPHL, subjects must use the drug for at least 24 weeks; however, in practice, most subjects use the drug for less than 30 days. The main reason for low compliance with topical minoxidil use is the poor cosmetic properties of the drug. ${ }^{5-6}$ To address these shortcomings, we have developed a novel 
"After Minoxidil" spray that removes excess minoxidil from hair while leaving minoxidil on the scalp.

A major limitation of this study was the small sample size. Nevertheless, the novel "After Minoxidil" spray improved ease of styling, hair manageability and reduces overall greasiness. Subjects using the "After Minoxidil" spray reported an increased willingness to adhere to a minoxidil treatment regimen for 90 days. To the best of our knowledge, this is the first cosmetic product that enhances minoxidil's cosmetic shortcomings and subsequently improves minoxidil compliance.

\section{References}

1. Gan DC and Sinclair RD. Prevalence of Male and Female Pattern Hair Loss in Maryborough. J Investig Dermatol Symp Proc. 2005; 10(3): 184-189.

2. US FDA Application 21-812 Medical Review

3. Mann D. Survey: Most Baldness Treatments Don't Work.

https://www.webmd.com/skin-problems-and-treatments/hair-loss/news/20100406/surveymost-baldness-treatments-dont-work\#1

4. Olsen EA, Whiting D, Bergfeld W, Miller J, Hordinsky M, Wanser R, et al. A multicenter, randomized, placebo-controlled, double-blind clinical trial of a novel formulation of 5\% minoxidil topical foam versus placebo in the treatment of androgenetic alopecia in men. J Am Acad Dermatol. 2007; 57(5): 767-74

5. Gupta AK, Charrette A. Topical Minoxidil: Systematic Review and Meta-Analysis of Its Efficacy in Androgenetic Alopecia. Skinmed. 2015; 13(3): 185-189.

6. Pereira MN, Schulte HL, Duarte N, Lima EM, Sá-Barreto LL, Gratieri T, Gelfuso GM, Cunha-Filho MS. Solid Effervescent Formulations as New Approach for Topical Minoxidil Delivery. Eur J Pharm Sci. 2017; 96: 411-419. 

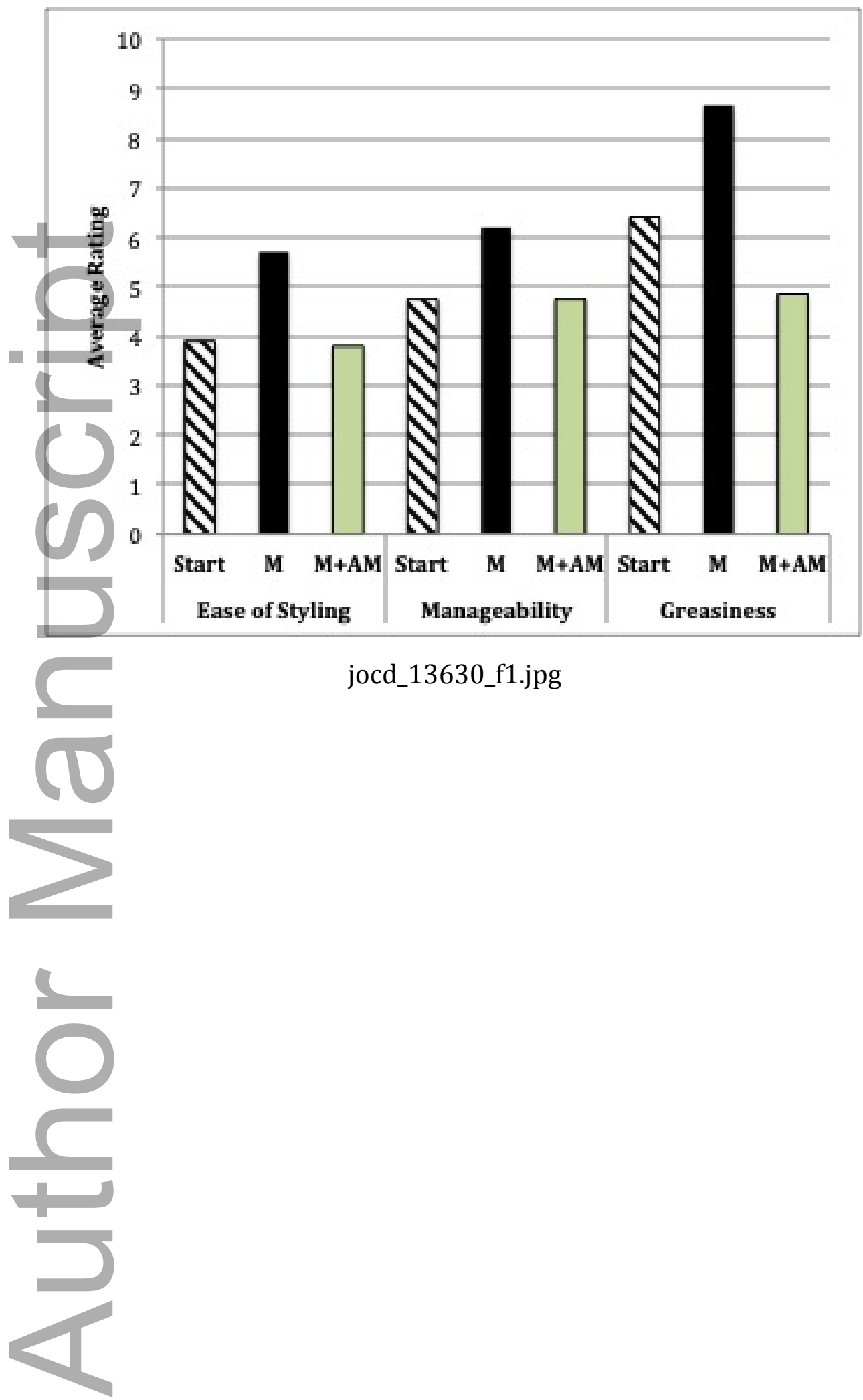

jocd_13630_f1.jpg 


\section{University Library}

\section{- M M I N E R VA A gateway to Melbourne's research publications}

Minerva Access is the Institutional Repository of The University of Melbourne

\section{Author/s:}

Kovacevic, M;McCoy, J;Shapiro, J;Sinclair, R;Vano-Galvan, S;Goldust, M;Situm, M;Goren, A

Title:

Novel "After Minoxidil" spray improves topical minoxidil compliance and hair style manageability

\section{Date:}

2020-08-26

\section{Citation:}

Kovacevic, M., McCoy, J., Shapiro, J., Sinclair, R., Vano-Galvan, S., Goldust, M., Situm, M. \& Goren, A. (2020). Novel "After Minoxidil" spray improves topical minoxidil compliance and hair style manageability. JOURNAL OF COSMETIC DERMATOLOGY, 19 (10), pp.2647-2649. https://doi.org/10.1111/jocd. 13630.

Persistent Link:

http://hdl.handle.net/11343/276190 